\title{
Estimation de la prévalence de la multimorbidité au moyen du Système canadien de surveillance des maladies chroniques
}

\author{
Allison Feely, B. Sc. (1); Lisa M. Lix, Ph. D. (2); Kim Reimer, B. Sc. (3)
}

Cet article a fait l'objet d'une évaluation par les pairs.

\section{Résumé}

Introduction : Le Système canadien de surveillance des maladies chroniques (SCSMC) de l'Agence de la santé publique du Canada utilise une méthode validée et normalisée pour estimer la prévalence des maladies chroniques, par exemple le diabète. L'élargissement de la portée du SCSMC pour inclure la surveillance de la multimorbidité et de la présence concomitante de deux maladies chroniques ou plus pourrait mieux guider la promotion de la santé et la prévention des maladies. L'objectif de notre étude était de déterminer s'il était possible de recourir au SCSMC pour estimer la prévalence de la multimorbidité.

Méthodologie : Nous avons utilisé les données administratives sur la santé de sept provinces et de trois territoires portant sur cinq affections chroniques validées (maladies cardiovasculaires, maladies respiratoires, maladies mentales, hypertension et diabète) pour estimer la prévalence de la multimorbidité. Nous avons produit des estimations normalisées selon l'âge et spécifiques selon l'âge (à l'aide des données démographiques canadiennes de 1991) pour deux définitions de la multimorbidité, soit deux affections ou plus ou trois affections ou plus sur les cinq affections validées, selon le sexe, la période et la zone géographique.

Résultats : Au cours de l'exercice 2011-2012, la prévalence d'au moins deux et d'au moins trois affections chroniques chez les Canadiens de 40 ans ou plus se situait à respectivement $26,5 \%$ et $10,2 \%$, ce qui est comparable à d'autres estimations faites à partir des données administratives sur la santé. L'augmentation de la prévalence de la multimorbidité avec l'âge était similaire dans toutes les provinces. Les différences de prévalence entre hommes et femmes variaient selon les provinces et territoires. Nous avons également observé une variation importante des estimations au fil des années. Les résultats obtenus étaient comparables pour les deux définitions de la multimorbidité.

Conclusion : La méthodologie du SCSMC permet de produire des estimations comparatives de la prévalence de la multimorbidité dans l'ensemble des provinces et des territoires, mais son utilisation pour estimer les variations temporelles pose des difficultés. L'augmentation du nombre et de la portée des définitions de cas validées dans le SCSMC permettra d'améliorer l'exactitude de la surveillance de la multimorbidité auprès de la population canadienne.

Mots-clés : maladies chroniques, surveillance, prévalence, SCSMC

\section{Introduction}

La multimorbidité, soit la présence concomitante de plusieurs affections chroniques dont l'une n'est pas nécessairement plus importante que les autres ${ }^{1}$, est de plus en plus fréquente, en particulier chez les personnes âgées ${ }^{2-7}$. On s'attend à une augmentation de la prévalence de la multimorbidité, au Canada comme dans d'autres pays, en raison du vieillissement de la population et d'une augmentation de la prévalence d'affections chroniques comme le diabète et l'hypertension ${ }^{8}$. La multimorbidité est

\section{Diffuser cet article sur Twitter}

\section{Points saillants}

- Le Système canadien de surveillance des maladies chroniques (SCSMC) utilise une méthodologie normalisée fondée sur les données administratives pour estimer la prévalence des maladies chroniques, par exemple le diabète, dans les provinces et les territoires. Nous avons examiné la possibilité de recourir au SCSMC pour assurer la surveillance de la multimorbidité, qui se définit généralement comme la présence concomitante de deux affections chroniques ou plus.

- La prévalence globale de la multimorbidité d'après cette définition était de 26,5 \% en 2011-2012, en utilisant les données relatives à cinq affections (maladies cardiovasculaires, maladies respiratoires, maladies mentales, hypertension et diabète) fournies par sept provinces et trois territoires. Les tendances spécifiques à l'âge étaient similaires dans l'ensemble des provinces et des territoires, mais des variations importantes ont été observées au fil du temps.

- Le SCSMC sera de plus en plus utile pour assurer la surveillance de la multimorbidité à l'échelle nationale, au fur et à mesure que de nouvelles définitions de cas de maladies chroniques y seront ajoutées.

un enjeu important pour les fournisseurs de soins de santé et les décideurs, car elle a été associée à des conséquences pour la santé potentiellement négatives, en particulier à une diminution de la qualité de vie liée à la santé ${ }^{9}$ et à une augmentation de

Rattachement des auteures :

1. Département de statistique, Université du Manitoba, Winnipeg (Manitoba), Canada

2. Département des sciences de la santé communautaire, Université du Manitoba, Winnipeg (Manitoba), Canada

3. Ministère de la Santé de la Colombie-Britannique, Victoria (Colombie-Britannique), Canada

Correspondance : Lisa M. Lix, George and Fay Yee Centre for Healthcare Innovation, Chown Building, $3^{\mathrm{e}}$ étage, 753, avenue McDermot, Winnipeg (Manitoba) R3E 0T6; tél. : 204-789-3573; télec. : 204-789-3905; courriel : Lisa.Lix@umanitoba.ca 
l'utilisation ainsi que des coûts des soins de santé ${ }^{10,11}$.

Le Système canadien de surveillance des maladies chroniques (SCSMC) est le fruit des efforts concertés de l'Agence de la santé publique du Canada (ASPC) et des gouvernements provinciaux et territoriaux. L'objectif du SCSMC est de produire des estimations exactes de la prévalence et de l'incidence d'affections chroniques comme le diabète et l'hypertension. Cette information peut être utilisée à plusieurs fins, notamment pour évaluer les répercussions des affections chroniques sur le système de santé. Le SCSMC produit des données comparatives au moyen d'une méthodologie axée sur la population qui a été validée et normalisée à l'échelle des provinces et des territoires. À l'heure actuelle, toutefois, le SCSMC s'intéresse principalement aux affections chroniques prises individuellement et son utilisation pour la surveillance de la multimorbidité n’a pas été étudiée.

Pour le moment, on dispose de peu de données à l'échelle de la population sur la multimorbidité au Canada. Roberts et ses collaborateurs ${ }^{7}$ ont utilisé les données de l'Enquête sur la santé dans les collectivités canadiennes (ESCC) pour estimer la prévalence de la multimorbidité pour une année et prouver l'existence de son association avec les déterminants de la santé comme l'âge et le revenu. Kuwornu et ses collaborateurs ${ }^{12}$ ont utilisé les données de l'ESCC pour comparer la prévalence et les caractéristiques de la multimorbidité chez les populations autochtones et les populations non autochtones d'origine blanche du Canada. Cependant, aucune étude axée sur la population n'a fourni d'estimations comparatives pour l'ensemble des territoires et des provinces du Canada. Quelques études axées sur la population ont été réalisées pour quelques provinces ou territoires pris individuellement ${ }^{6,13,14}$, mais une seule ${ }^{6}$ d'entre elles a examiné les variations de la multimorbidité au fil du temps, et aucune n'a examiné les variations au sein de sousgroupes de population. Dans ce contexte, l'objet de notre étude consistait à évaluer la possibilité de recourir au SCSMC pour estimer la prévalence de la multimorbidité au sein de groupes de la population définis selon l'âge, le sexe et la zone géographique, ainsi qu'au fil du temps.

\section{Méthodologie}

\section{Sources de données}

$\mathrm{Au}$ total, 10 provinces et territoires ont fourni des données pour les analyses dont il est question dans cette étude : la Colombie-Britannique, le Manitoba, l'Ontario, le Québec, le Nouveau-Brunswick, la Nouvelle-Écosse, Terre-Neuve-et-Labrador et le Nunavut. Ces provinces et territoires ont répondu à l'appel de données pour la version 2015 du SCSMC lancé en avril 2015. Ils rassemblent environ $86 \%$ de la population canadienne totale, et toutes les populations du Nord du Canada ${ }^{15}$.

Au nombre des bases de données administratives sur la santé utilisées pour estimer la prévalence de la multimorbidité figurent les dossiers des hôpitaux, les données de facturation des médecins et le registre de la population. Les dossiers des hôpitaux et les données de facturation des médecins fournissent des renseignements sur les cas diagnostiqués de maladies en respectant la Classification internationale des maladies, neuvième révision (CIM-9) ${ }^{16}$, la Classification internationale des maladies, neuvième révision, modification clinique (ICD-9-MC) ${ }^{17}$ et la Classification statistique internationale des maladies et des problèmes de santé connexes, dixième révision, Canada (CIM$10-\mathrm{CA})^{18}$. Le registre de la population recueille les données de tous les résidents des provinces et des territoires ayant une couverture d'assurance-maladie valide et fournit également des données démographiques (âge et sexe). Ces trois sources de données peuvent être liées de façon anonyme au moyen d'un identificateur unique valide à vie (numéro d'assurancemaladie).

\section{Définition des affections chroniques sélectionnées}

Notre étude porte sur cinq affections chroniques : 1) les maladies cardiovasculaires, qui incluent la cardiopathie ischémique et l'insuffisance cardiaque; 2) les maladies respiratoires, qui incluent l'asthme et les maladies pulmonaires obstructives chroniques (MPOC); 3) les maladies mentales, une catégorie générale du SCSMC (codes 290 à 319 de la CIM-9) qui englobe la psychose, les troubles névrotiques, les troubles de la personnalité, d'autres troubles mentaux non psychotiques et le retard mental; 4) l'hypertension et 5) le diabète. Nous avons choisi ces affections chroniques parce qu'elles étaient associées à des définitions de cas validées du SCSMC ${ }^{19-25}$. D'autres affections chroniques fréquentes chez les adultes de 40 ans et plus, comme l'arthrite et l'ostéoporose, sont incluses dans d'autres définitions de la multimorbidité, mais ne comportaient pas de définitions de cas validées du SCSMC au moment de la réalisation de notre étude. Toutes les affections chroniques sélectionnées avaient déjè été incluses dans des recherches antérieures sur la mesure de la multimorbidité26.

Les affections chroniques ont été sélectionnées au moyen de règles de définition des cas (tableau 1) appliquées aux données administratives des exercices 1995-1996 et ultérieurs (un exercice s'échelonne du $1^{\text {er }}$ avril au 31 mars) et les estimations de la prévalence ont été produites pour les exercices 2001-2002 et 2011-2012. Chaque règle relative à un cas, élaborée par un groupe de travail du SCSMC, décrit le nombre et les types de codes de diagnostic qui doivent être consignés dans une base de données administrative au cours d'une période définie pour qu'une personne soit considérée comme un cas de maladie. L'exercice 2011-2012 était l'exercice le plus récent pour lequel des données étaient disponibles au moment où l'appel de données a été lancé aux provinces et aux territoires.

Nous avons évalué deux définitions de la multimorbidité. La première, la plus courante, tient compte de la présence concomitante de deux affections chroniques ou plus. La seconde est basée sur la présence concomitante de trois affections chroniques ou plus. Cette définition a déjà été étudiée dans le cadre de travaux de recherche antérieurs ${ }^{7}$.

\section{Analyse statistique}

Nous avons estimé la prévalence de la multimorbidité chez les personnes de 40 ans et plus selon le sexe, l'âge (tranches d'âge de cinq ans), la province ou le territoire, la définition et la période (année d'exercice). Nous avons fixé l'âge minimum à 40 ans car il s'agit de la limite d'âge inférieure commune aux définitions de cas d'affections chroniques incluses dans cette étude. Nous avons calculé les taux de prévalence normalisés selon l'âge, les taux de prévalence spécifiques selon l'âge et les taux de prévalence bruts pour chaque province et territoire ainsi que pour l'ensemble des 10 provinces et territoires combinés. La population canadienne de 1991 a servi de référence pour la normalisation selon l'âge. Nous avons calculé les taux de prévalence bruts en divisant le nombre de personnes avec multimorbidité par la population totale définie à partir du registre de l'état civil provincial ou territorial. Les chiffres 
TABLEAU 1

Définitions de cas du SCSMC des maladies chroniques sélectionnées pour estimer la prévalence de la multimorbidité

\begin{tabular}{|c|c|c|c|c|c|c|}
\hline \multirow[t]{2}{*}{ Maladies chroniques } & \multirow[t]{2}{*}{ Algorithme } & \multirow{2}{*}{$\begin{array}{l}\text { Groupe } \\
\text { d'âge } \\
\text { (ans) }\end{array}$} & \multirow{2}{*}{$\begin{array}{c}\text { Date d'identification } \\
\text { du cas }\end{array}$} & \multicolumn{2}{|c|}{$\begin{array}{l}\text { Codes inscrits au fichier des } \\
\text { hospitalisations et au fichier } \\
\text { de facturation des médecins }\end{array}$} & \multirow[t]{2}{*}{ Exclusions } \\
\hline & & & & CIM-9-MC & CIM-10-CA & \\
\hline \multicolumn{7}{|l|}{ Cardiovasculaires } \\
\hline Cardiopathie ischémique & $\begin{array}{l}\text { Au moins une hospitalisation ou au } \\
\text { moins deux diagnostics consignés au } \\
\text { fichier de facturation des médecins au } \\
\text { cours d'une période d'un an }\end{array}$ & $20+$ & $\begin{array}{l}\text { Sortie de l'hôpital ou } \\
\text { dernière consultation } \\
\text { (selon la première } \\
\text { éventualité) }\end{array}$ & $410-414$ & $120-125$ & Aucune \\
\hline Insuffisance cardiaque & $\begin{array}{l}\text { Au moins une hospitalisation ou au } \\
\text { moins deux diagnostics consignés au } \\
\text { fichier de facturation des médecins au } \\
\text { cours d'une période d'un an }\end{array}$ & $40+$ & $\begin{array}{l}\text { Sortie de l'hôpital ou } \\
\text { dernière consultation } \\
\text { (selon la première } \\
\text { éventualité) }\end{array}$ & 428 & 150 & Aucune \\
\hline \multicolumn{7}{|l|}{ Respiratoires } \\
\hline MPOC & $\begin{array}{l}\text { Au moins une hospitalisation ou au } \\
\text { moins un diagnostic consigné au } \\
\text { fichier de facturation des médecins }\end{array}$ & $35+$ & $\begin{array}{l}\text { Sortie de l'hôpital ou } \\
\text { dernière consultation } \\
\text { (selon la première } \\
\text { éventualité) }\end{array}$ & 491, 492, 496 & $\mathrm{~J} 41-\mathrm{J} 44$ & Aucune \\
\hline \multicolumn{7}{|l|}{ Maladies mentales } \\
\hline $\begin{array}{l}\text { Ensemble des maladies } \\
\text { mentales }\end{array}$ & $\begin{array}{l}\text { Au moins une hospitalisation ou au } \\
\text { moins un diagnostic consigné au } \\
\text { fichier de facturation des médecins au } \\
\text { cours d'une période d'un an }\end{array}$ & $0+$ & $\begin{array}{l}\text { Sortie de l'hôpital ou } \\
\text { dernière consultation } \\
\text { (selon la première } \\
\text { éventualité) }\end{array}$ & $290-319$ & F00-F99 & Aucune \\
\hline \multicolumn{7}{|l|}{ Hypertension } \\
\hline & $\begin{array}{l}\text { Au moins une hospitalisation ou au } \\
\text { moins deux diagnostics consignés au } \\
\text { fichier de facturation des médecins au } \\
\text { cours d'une période de deux ans }\end{array}$ & $20+$ & $\begin{array}{l}\text { Sortie de l'hôpital ou } \\
\text { dernière consultation } \\
\text { (selon la première } \\
\text { éventualité) }\end{array}$ & $401-405$ & $\mathrm{I10-I13,} \mathrm{I15}$ & $\begin{array}{l}\text { Hypertension } \\
\text { gravidique chez } \\
\text { les femmes de } \\
20 \text { à } 54 \text { ans }\end{array}$ \\
\hline
\end{tabular}

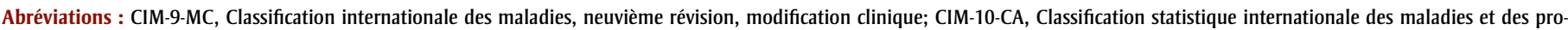
blèmes de santé connexes, dixième révision, Canada; MPOC, maladie pulmonaire obstructive chronique; SCSMC, Système canadien de surveillance des maladies chroniques.

ayant servi au calcul de la prévalence ont été arrondis, de façon classique, au multiple de cinq près (à la dizaine près dans le cas de l'Ontario et des données pour l'ensemble des provinces et des territoires).

Nous avons présenté les données sous forme de tableaux et de graphiques. Les comparaisons entre les provinces et territoires au fil du temps et entre les sousgroupes de population ont été effectuées en fonction des pourcentages, des rangs et du coefficient de variation, qui est une mesure de la dispersion statistique. Nous avons produit des estimations de l'ampleur de l'écart entre les sous-groupes en utilisant la distribution du chi carré $\left(\chi^{2}\right)$ pour les échantillons de grande taille, avec un intervalle de confiance à $95 \%$ (IC à $95 \%$ ). Nous avons eu recours au coefficient de corrélation de rang de Spearman pour décrire l'association entre les estimations de la prévalence obtenues à l'aide des deux définitions de la multimorbidité à l'échelle provinciale ou territoriale, étant donné que nous ne pouvions pas supposer que les estimations suivraient une courbe de distribution normale. La méthode statistique non paramétrique de Mantel-Haenszel, qui suit, de façon asymptotique, la courbe de distribution $\mathrm{du} \chi^{2}$, a été utilisée pour mesurer la déviation par rapport à la tendance linéaire au fil du temps. Toutes les analyses statistiques ont été effectuées à l'aide du SAS version $9.3^{27}$.

\section{Résultats}

Le tableau 2 présente l'estimation de la prévalence de la multimorbidité normalisée selon l'âge pour chaque définition de la multimorbidité (deux affections concomitantes ou plus, et trois affections concomitantes ou plus), pour chaque province et territoire ainsi que pour l'ensemble des 10 provinces et territoires, au cours du premier et du dernier exercice de la période à l'étude. En 2011-2012, la prévalence globale normalisée selon l'âge de deux affections 
TABLEAU 2

Estimations (\%) de la prévalence de la multimorbidité normalisée selon l'âgé et IC à $95 \%$, stratifiés selon la définition de la multimorbidité et la période

\begin{tabular}{|c|c|c|c|c|c|c|c|}
\hline \multirow{4}{*}{$\begin{array}{l}\text { Province ou } \\
\text { territoire } \\
\text { C.-B. }\end{array}$} & \multicolumn{7}{|c|}{ Définition de la multimorbidité (nombre d'affections chroniques) } \\
\hline & \multicolumn{3}{|c|}{ Deux affections ou plus } & \multicolumn{4}{|c|}{ Trois affections ou plus } \\
\hline & \multirow{2}{*}{$\begin{array}{c}\begin{array}{c}2001-2002 \\
\%(\text { (IC à } 95 \%)\end{array} \\
17,4 \quad(17,4-17,5)\end{array}$} & $\begin{array}{c}2011-2012 \\
\% \text { (IC à } 95 \%)\end{array}$ & Hausse en \% (rang) & \multicolumn{2}{|c|}{$\begin{array}{c}\text { 2001-2002 } \\
\text { \% (IC à } 95 \% \text { ) }\end{array}$} & $\begin{array}{c}2011-2012 \\
\text { \% (IC à } 95 \%)\end{array}$ & $\begin{array}{l}\text { Hausse en } \% \\
\quad \text { (rang) }\end{array}$ \\
\hline & & $24,8(24,8-24,9)$ & $42,5 \quad(3)$ & 5,2 & $(5,1-5,2)$ & $9,1 \quad(9,1-9,2)$ & 75,0 \\
\hline Man. & $20,4(20,3-20,5)$ & $27,7 \quad(27,6-27,8)$ & $35,8 \quad(5)$ & 6,4 & $(6,3-6,4)$ & $10,3 \quad(10,3-10,4)$ & $60,9 \quad(5)$ \\
\hline N.-B. & $19,6(19,5-19,8)$ & $27,5 \quad(27,4-27,7)$ & $40,3 \quad(4)$ & 6,5 & $(6,4-6,5)$ & $10,4 \quad(10,3-10,5)$ & 60,0 \\
\hline N.-É. & $23,5 \quad(23,4-23,6)$ & $30,3 \quad(30,1-30,4)$ & $28,9 \quad(7)$ & 7,8 & $(7,7-7,8)$ & $11,8 \quad(11,7-11,9)$ & $51,3 \quad(7)$ \\
\hline T.-N.-L. & $22,5 \quad(22,3-22,7)$ & $28,1 \quad(27,9-28,3)$ & $24,9 \quad(10)$ & 7,3 & $(7,2-7,5)$ & $10,2 \quad(10,1-10,3)$ & $39,7 \quad(10)$ \\
\hline Yn & $19,3 \quad(18,3-20,2)$ & $27,6 \quad(26,8-28,5)$ & $43,0 \quad(2)$ & 6,1 & $(5,6-6,7)$ & $10,9 \quad(10,4-11,5)$ & $78,7 \quad(2)$ \\
\hline
\end{tabular}

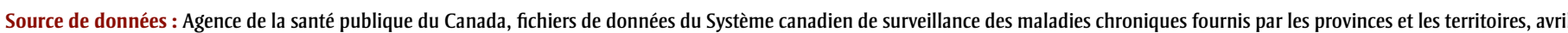
2015. Données non disponibles pour l'Alberta, la Saskatchewan et l'île-du-Prince-Édouard.

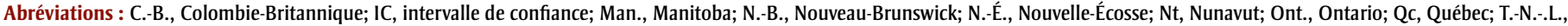
Terre-Neuve-et-Labrador; T.N.-O., Territoires du Nord-Ouest; Yn, Yukon.

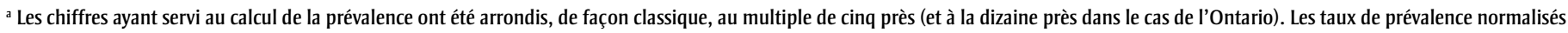
selon l'âge ont été calculés à l'aide de données non arrondies sur la prévalence.

chroniques ou plus était de $26,5 \%$, soit une augmentation relative de $29,3 \%$ par rapport à l'estimation de 2001-2002, qui se situait à 20,5 \%. La prévalence globale normalisée selon l'âge de trois affections chroniques ou plus était de 10,2 \% en 20112012, ce qui représente une augmentation de $50 \%$ par rapport à l'estimation de 6,8 \% en 2001-2002. La tendance linéaire de la prévalence de deux affections ou plus était statistiquement significative $(p<0,001)$, de même pour la prévalence de trois affections ou plus $(p<0,001)$. Une forte corrélation a été observée entre les estimations de la prévalence obtenues pour les deux définitions de la multimorbidité à l'échelle provinciale ou territoriale au moyen du coefficient de corrélation de Spearman, avec un taux de corrélation estimé de 0,94 en 2001-2002 (données non illustrées).

Dans le cas de la définition de la multimorbidité liée à la présence de deux affections chroniques ou plus, l'estimation de la prévalence la plus faible parmi les provinces et les territoires était de 6,5\% (Nunavut) en 2001-2002 et de 24,0 \% (Nunavut) en 2011-2012. L'estimation de la prévalence la plus élevée, observée en Nouvelle-Écosse, était de $23,5 \%$ en 20012002 et de 30,3 \% en 2011-2012. Dans le cas de la définition de la multimorbidité liée à la présence de trois affections chroniques ou plus, l'estimation de la prévalence la plus faible était de 1,4\% (Nunavut) en 2001-2002 et de 9,1 \% (Colombie-Britannique) en 2011-2002. L'estimation de la prévalence la plus élevée était de 7,8\% (Nouvelle-Écosse) en 2000-2002 et de $12,0 \%$ (Nunavut) en 2011-2012. Le classement des provinces et des territoires en ce qui concerne la hausse de pourcentage entre les exercices 2001-2002 et 20112012 était similaire pour les deux définitions de la multimorbidité. Le Nunavut a présenté la plus forte augmentation de la prévalence, soit $326,2 \%$ pour la présence de deux affections chroniques ou plus et $757,1 \%$ pour la présence de trois affections chroniques ou plus entre ces deux périodes. La plus faible augmentation de la prévalence a été observée à Terre-NeuveLabrador, soit $24,9 \%$ pour la présence de deux affections chroniques ou plus et $39,7 \%$ pour la présence de trois affections chroniques ou plus.

La figure 1 montre la prévalence normalisée selon l'âge de deux affections chroniques concomitantes ou plus selon le sexe et la province ou territoire en 2011-2012. Chez les hommes, la prévalence globale était de $1,1 \%$ (IC à $95 \%: 1,1$ à 1,2 ) supérieure à celle observée chez les femmes. La prévalence était plus élevée chez les hommes que chez les femmes dans plusieurs provinces. En revanche, la prévalence était plus élevée chez les femmes que chez les hommes dans tous les territoires. L'écart absolu le plus faible dans l'estimation de la prévalence entre les hommes et les femmes a été observé à Terre-Neuve-Labrador (0,1 \%). L'écart absolu le plus élevé a quant à lui été observé au Nunavut $(3,8 \%)$. Chez les hommes, la prévalence globale de trois affections chroniques ou plus était de 1,4\% (IC à $95 \%$ : 1,3 à 1,4) supérieure à celle observée chez les femmes et si, dans les provinces, la prévalence était plus élevée chez les hommes que chez les femmes, dans les territoires, elle était en revanche plus élevée chez les femmes que chez les hommes (données non illustrées).

La figure 2 présente la prévalence spécifique selon l'âge de deux affections chroniques concomitantes ou plus pour chaque province et territoire en 2011-2012. La prévalence globale observée dans le groupe le plus âgé (85 ans et plus) était de $66,3 \%$. Ce taux était $58,6 \%$ plus élevé que la prévalence globale observée dans le groupe 
FIGURE 1

Prévalence $^{\mathrm{a}}(\%)$ normalisée selon l'âge de deux maladies chroniques concomitantes ou plus chez les personnes de 40 ans et plus, selon le sexe et la province ou le territoire, 2011-2012

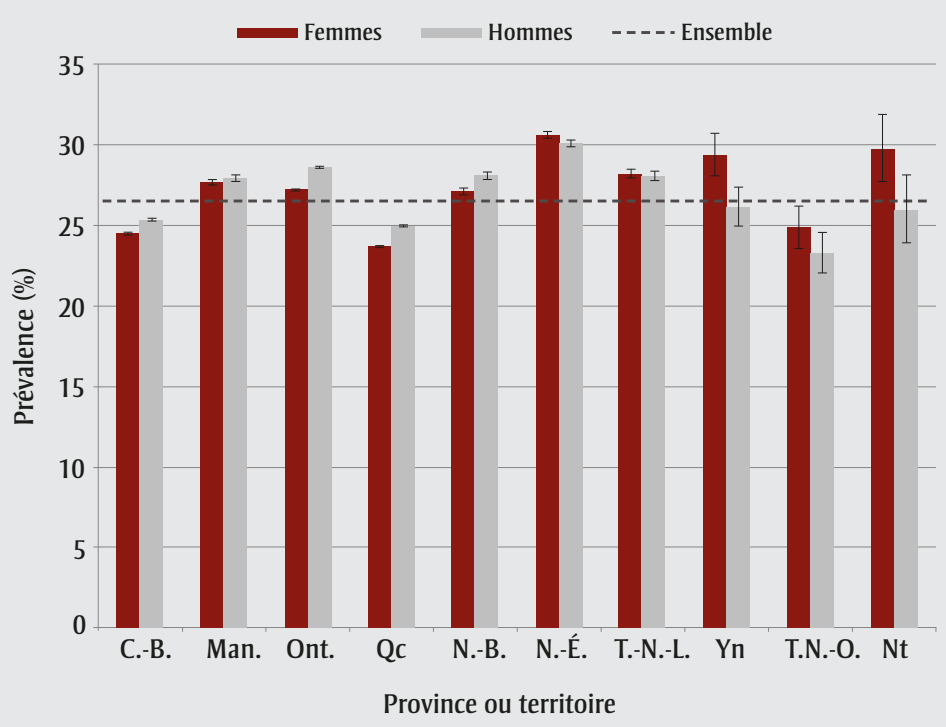

Source de données : Agence de la santé publique du Canada, fichiers de données du Système canadien de surveillance des mala dies chroniques fournis par les provinces et les territoires, avril 2015. Données non disponibles pour l'Alberta, la Saskatchewan et l'île-du-Prince-Édouard.

Abréviations : C.-B., Colombie-Britannique; Man., Manitoba; N.-B., Nouveau-Brunswick; N.-É., Nouvelle-Écosse; Nt, Nunavut Ont., Ontario; Qc, Québec; T.-N.-.L., Terre-Neuve-et-Labrador; T.N.-O., Territoires du Nord-Ouest; Yn, Yukon.

Remarque : I désigne un intervalle de confiance à $95 \%$.

a Les chiffres ayant servi au calcul de la prévalence ont été arrondis, de façon classique, au multiple de cinq près (et à la dizaine près dans le cas de l'Ontario). Les taux de prévalence normalisés selon l'âge ont été calculés à l'aide de données non arrondies sur la prévalence.

FIGURE 2

Prévalence $^{\mathrm{a}}(\%)$ de deux maladies chroniques concomitantes ou plus, selon le groupe d'âge et la province ou le territoire, 2011-2012

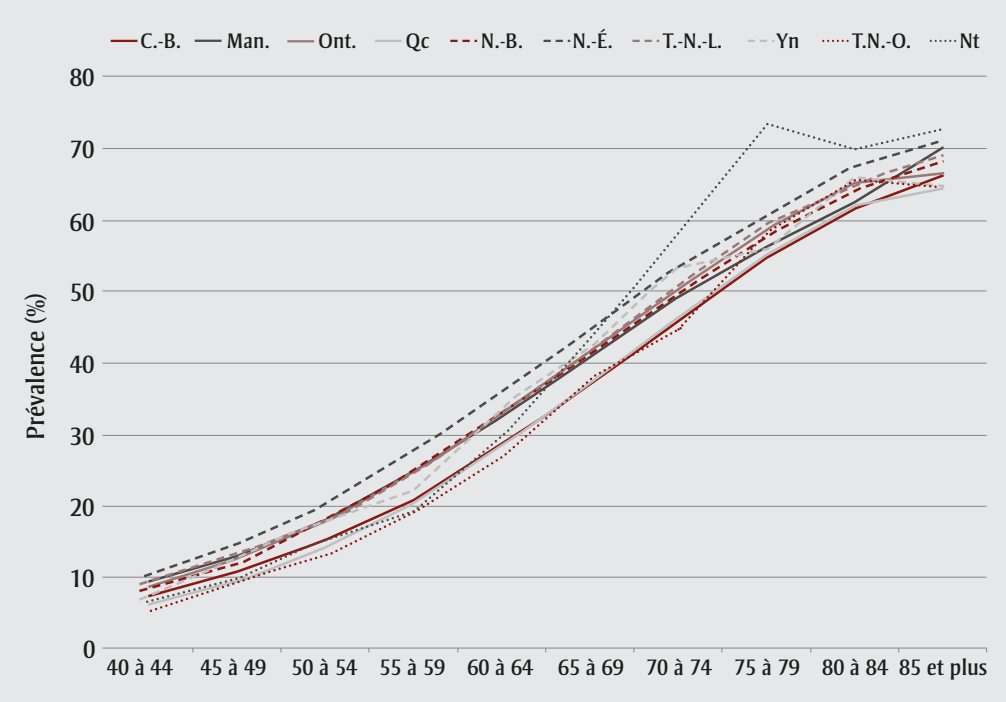

Groupe d'âge (ans)

Source de données : Agence de la santé publique du Canada, fichiers de données du Système canadien de surveillance des mala dies chroniques fournis par les provinces et les territoires, avril 2015. Données non disponibles pour l'Alberta, la Saskatchewan et l'île-du-Prince-Édouard.

Abréviations : C.-B., Colombie-Britannique; Man., Manitoba; N.-B., Nouveau-Brunswick; N.-É., Nouvelle-Écosse; Nt, Nunavut; Ont., Ontario; Qc, Québec; T.-N.-.L., Terre-Neuve-et-Labrador; T.N.-O., Territoires du Nord-Ouest; Yn, Yukon.

${ }^{a}$ Les chiffres ayant servi au calcul de la prévalence ont été arrondis, de façon classique, au multiple de cinq près (et à la dizaine près dans le cas de l'Ontario). Les taux de prévalence normalisés selon l'âge ont été calculés à l'aide de données non arrondies sur la prévalence. des personnes les plus jeunes (40 à 44 ans; 7,8 \%). En 2001-2002 (données non illustrées), la prévalence globale était de $5,5 \%$ dans le groupe le plus jeune et de 52,1\% dans le groupe le plus âgé. En 2011-2012, la prévalence globale de trois affections chroniques ou plus était de $1,4 \%$ dans le groupe le plus jeune et de 35,6\% dans le groupe le plus âgé (données non illustrées).

Pour l'ensemble des groupes d'âge, la tendance suit une courbe en « $\mathrm{S}$ » pour toutes les provinces et tous les territoires. En 2001-2002, le coefficient de variation pour les provinces et les territoires était similaire dans l'ensemble des groupes d'âge : de 0,28 chez 40 à 44 ans et de 0,27 chez les 85 ans et plus. En 2011-2012, le coefficient de variation était de 0,24 dans le groupe le plus jeune et de 0,14 , soit à peine plus bas, dans le groupe le plus âgé. Une tendance similaire a été observée en ce qui concerne la prévalence de trois affections chroniques ou plus, en ce sens que le coefficient de variation pour 2011-2012 était plus élevé chez le groupe le plus jeune $(0,72)$ et moins élevé chez le groupe le plus âgé $(0,30)$. En ce qui concerne la prévalence de trois affections chroniques ou plus, le coefficient de variation pour 2001-2002 était de 0,57 chez le groupe le plus jeune et de 0,20 chez le groupe le plus âgé.

\section{Analyse}

Chez la population de 40 ans et plus des 10 provinces et territoires ayant soumis des données d'étude au SCSMC, environ le quart présentait au moins deux des cinq affections chroniques validées et environ $10 \%$ présentaient au moins trois des cinq affections chroniques validées pour lesquelles les données du SCSMC avaient été recueillies. Notre estimation globale de 26,5\% (pour la présence de deux affections ou plus) en 2011-2012 est plus faible que celle d'une étude récente dans laquelle on estimait la prévalence de la multimorbidité au Canada à 42,6 \% chez la population âgée de 18 ans ou plus ${ }^{28}$ à partir des données du système national des dossiers médicaux électroniques. Fortin et ses collaborateurs $^{29}$ ont observé que les estimations de la prévalence de la multimorbidité établies pour les populations ayant recours à des soins primaires étaient souvent plus élevées que celles établies pour la population générale. De plus, cette étude a utilisé une liste de 20 affections chroniques pour recenser les patients atteints de multimorbidités, alors que notre étude a utilisé une liste de cinq affections chroniques seulement. 
Au moyen des données de l'ESCC de 20112012, Roberts et ses collaborateurs ${ }^{7}$ ont estimé que la prévalence, à l'échelle nationale, de deux affections chroniques ou plus était de $12,9 \%$, et que la prévalence de trois affections chroniques ou plus était de $3,9 \%$, soit des estimations significativement plus faibles que les nôtres, et qui pourraient mettre en évidence les effets du biais lié à l'autodéclaration sur la mesure des maladies chroniques ${ }^{30}$. L'écart entre les estimations pourrait également s'expliquer en partie par la différence liée aux groupes d'âge étudiés : Roberts et ses collaborateurs ${ }^{7}$ ont inclus les personnes de 20 ans et plus, tandis que nous n'avons estimé la prévalence de la multimorbidité que chez les personnes de 40 ans et plus. Une étude menée en Ontario ${ }^{6}$ s'appuyant sur des données administratives sur la santé pour estimer la prévalence de la multimorbidité (deux affections ou plus) a fait état d'une prévalence de $24,3 \%$ en 2009 . Cependant, elle incluait un éventail plus vaste d'affections chroniques (16 au total) que celles incluses dans l'étude du SCSMC et portait également sur un intervalle d'âge plus étendu (de la naissance à 105 ans).

Nous n'avons observé, en utilisant les données du SCSMC, aucune tendance uniforme dans les différences entre les hommes et les femmes pour l'ensemble des provinces et territoires. Des recherches antérieures ont montré que l'ampleur des différences observées entre les hommes et les femmes s'explique elle aussi par le choix des affections utilisées pour mesurer la multimorbidité. ${ }^{31}$

Nous avons constaté que la prévalence normalisée selon l'âge de la multimorbidité augmentait considérablement au fil du temps. À ce jour, on n'a publié aucune étude longitudinale sur la prévalence de la multimorbidité au Canada à laquelle nous pourrions comparer nos résultats. De fait, il y a eu très peu d'études internationales qui ont examiné les tendances longitudinales relatives à la prévalence de la multimorbidité. L'étude menée par Uijen et van de Lisdonk $^{32}$ - et qui demeure une exception - a utilisé les données électroniques sur les soins primaires des Pays-Bas et a révélé que la prévalence de la multimorbidité avait doublé en 20 ans. Nos résultats montrent des augmentations de l'ordre de 25,2\% à $78,7 \%$ en 11 ans pour l'ensemble des provinces et des territoires étudiés, à l'exception du Nunavut. D'autres études sont nécessaires pour déterminer la cause de ces augmentations. Wong et ses collaborateurs ${ }^{33}$ affirment qu'il est possible qu'un nombre accru de cas faussement positifs s'accumule au fil du temps, ce qui pourrait contribuer à une surestimation des taux de prévalence croissante au fil des années étudiées. Dans le cas du Nunavut, les fortes augmentations de la prévalence pourraient s'expliquer par le fait que le Nunavut est officiellement devenu un territoire en 1999 et que, par conséquent, ses bases de données administratives n'ont pas eu suffisamment de temps pour saisir les données sur les cas prévalents avant l'exercice 2001-2002. Autrement dit, il est fort probable que l'on ait sous-estimé la prévalence pour la première année de l'étude comparativement à d'autres provinces où les données administratives de l'exercice 1995-1996 et des suivants ont été utilisées pour la détermination des cas.

\section{Forces et limites}

Au nombre des principales forces de cette étude figurent l'utilisation de la méthodologie normalisée et validée du SCSMC et la production d'estimations de la prévalence de la multimorbidité pour plus de $80 \%$ de la population canadienne adulte de 40 ans et plus. Une limite, toutefois, est que notre étude est fondée sur des définitions de cas validées qui sont associées à des affections chroniques individuelles plutôt que sur une définition de cas validée globale de la multimorbidité, et qu'elle se limite à cinq affections, celles définies au moment de l'appel de données lancé aux provinces et aux territoires. Fortin et ses collaborateurs ${ }^{31}$ ont fait observer que le fait de limiter les affections à moins de sept maladies chroniques pourrait donner lieu à une sousestimation de la prévalence de la multimorbidité, et ils ont recommandé la prise en compte de 12 maladies chroniques ou plus. Diederichs et ses collaborateurs ${ }^{34}$ ont sélectionné 11 affections qu'ils recommandent d'inclure dans les études sur la multimorbidité. Le diabète, la dépression, l'hypertension, les cardiopathies et les MPOC figurent dans leur liste, tout comme dans notre étude. D'autres affections, comme l'arthrite, les accidents vasculaires cérébraux, le cancer et l'ostéoporose, qui figurent dans d'autres définitions, n'avaient pas été validées par une définition de cas du SCSMC au moment de l'appel de données. Depuis, des travaux d'élaboration de définitions de cas pour bon nombre de ces affections ont été entamés ou sont achevés.

Le tableau 3 résume les forces et les faiblesses associées à l'utilisation du SCSMC pour estimer la prévalence de la multimorbidité. La méthodologie du SCSMC facilite les comparaisons entre les principaux déterminants de la santé, en particulier l'âge, le sexe et la région. Ces comparaisons sont utiles pour décrire les répercussions absolues et relatives de la multimorbidité sur différents groupes de population et elles peuvent aider à cibler des activités de promotion de la santé et de prévention des maladies. Cependant, l'utilisation SCSMC et des données administratives sur la santé pour mesurer la prévalence de la multimorbidité présente certains défis. À l'heure actuelle, aucune méthode ne permet de faire des comparaisons au moyen d'autres déterminants de la santé importants, comme le statut socioéconomique $\mathrm{e}^{7,12,13}$. Les données administratives dans lesquelles sont consignés les diagnostics peuvent comporter des erreurs de classification, ce qui peut introduire un biais dans les estimations de la prévalence ${ }^{35,36}$. Par ailleurs, les données administratives ne prennent pas en compte les personnes qui n'ont pas fait appel au système de soins de santé pour le traitement de leurs affections chroniques.

De plus, la conclusion selon laquelle la prévalence de la multimorbidité a augmenté au fil du temps pourrait s'expliquer, du moins en partie, par des changements dans la qualité et la disponibilité des données administratives sur la santé dans les provinces et les territoires. Les taux de prévalence au fil du temps peuvent également être influencés par la prise en compte de personnes ayant reçu à tort un ou plusieurs diagnostics de maladies chroniques ${ }^{6}$. En outre, les provinces et les territoires qui utilisent un seul code de diagnostic dans les données de facturation des médecins pourraient sous-estimer la prévalence de la multimorbidité, car la probabilité que plusieurs codes de diagnostics soient pris en compte dans ces données est alors réduite $^{37}$. Enfin, il convient de mentionner que les données administratives ne fournissent pas d'information sur la gravité des affections chroniques.

\section{Conclusion}

Nous avons utilisé des méthodes reconnues de surveillance des affections chroniques individuelles pour fournir des estimations comparatives de la multimorbidité dans divers provinces et territoires sur plus d'une décennie. Nos résultats révèlent plusieurs tendances conformes aux résultats de travaux de recherche 


\section{TABLEAU 3 \\ Forces et faiblesses liées à l'utilisation du SCSMC pour estimer la prévalence de la multimorbidité au Canada}

\section{Forces}

- Le SCSMC utilise une méthodologie uniformisée et validée dans toutes les provinces et les territoires.

- Le SCSMC utilise des données administratives sur la santé faisant l'objet d'une collecte systématique.

- L'utilisation des données du SCSMC permet de faire des comparaisons selon l'âge, le sexe, la région et la période visée.

- Le fait de réaliser des recherches à l'aide de données administratives sur la santé est moins coûteux que de procéder à la collecte de données primaires ${ }^{36,40}$.

- Les données du SCSMC ne sont pas influencées par le biais de rappel.

\section{Faiblesses}

- À l'heure actuelle, la méthodologie ne permet pas de faire des comparaisons au moyen de certains déterminants de la santé importants, en particulier le statut socioéconomique et l'origine ethnique.

- Les données administratives dans lesquelles sont consignés les diagnostics peuvent comporter des erreurs de classification ${ }^{34,35}$.

- Le SCSMC ne renferme pas d'information sur les résultats de laboratoire, qui permettraient de réduire les erreurs de classification, ni d'information sur les facteurs de risque de maladie chronique liés au mode de vie ( $\mathrm{p}$. ex. activité physique, tabagisme, etc.), qui pourraient à leur tour influer sur le risque de multimorbidités ${ }^{6,40}$.

- Le SCSMC ne prend pas en compte les personnes qui n'ont pas encore reçu de diagnostic pour une maladie chronique et qui font l'objet d'une investigation.

- À l'heure actuelle, un nombre limité d'affections chroniques validées sont incluses dans la méthodologie du SCSMC.

Abréviation : SCSMC, Système canadien de surveillance des maladies chroniques.

antérieurs, notamment des augmentations de la multimorbidité tout au long de la $\mathrm{vie}^{2-7}$. Bien qu'aucune tendance uniforme n’ait été mise en évidence dans l'ensemble des provinces et des territoires, nous avons observé une tendance à la hausse dans l'est du Canada par rapport à l'ouest, ce qui n'est pas étonnant à la lumière des résultats d'études antérieures ${ }^{38,39}$, ce qui semble indiquer que nos estimations ont une validité apparente. En ce qui concerne les augmentations de la prévalence au fil du temps, il existe peu d'études auxquelles nous pouvons comparer la nôtre, et aucune n'a été réalisée avec des données canadiennes, ce qui fait que ces estimations des tendances doivent être interprétées avec prudence.

Nous avons montré qu'il était possible de recourir au SCSMC pour le recensement des cas d'affections chroniques en vue de produire des estimations de la prévalence de la multimorbidité. Sa portée devrait toutefois être élargie, de manière à intégrer d'autres définitions de cas d'affections chroniques reconnues afin de dresser un portrait plus complet de la prévalence de la multimorbidité au Canada.

\section{Remerciements}

Cette étude a pu être réalisée grâce à la collaboration de l'Agence de la santé publique du Canada et des gouvernements de la Colombie-Britannique, du Manitoba, de l'Ontario, du Québec, du Nouveau-Brunswick, de la Nouvelle-Écosse, de Terre-Neuve-etLabrador, du Yukon, des Territoires du Nord-Ouest et du Nunavut. Les opinions, les résultats et les conclusions de l'étude n'engagent cependant que les auteures. On ne peut en déduire qu'ils ont été approuvés par les provinces et les territoires.

Si certaines sections du présent document sont fondées en partie sur l'information et les données compilées et fournies par l'Institut canadien d'information sur la santé (ICIS), les analyses et les conclusions présentées, ainsi que les opinions et les déclarations contenues dans cet article, sont celles des auteures et ne reflètent pas nécessairement la position de l'ICIS.

Nous aimerions également remercier les coprésidents et les membres du Comité scientifique du SCSMC pour leur participation à cette étude : Jay Onysko, Jean-François
Godin, Michael Paterson, Chris Waters, Larry Svenson, Carla Ens, Nancy Yu, Kimberly Blinco, Maurice Collette, John Knight, Yalda Jarfari, Heather Hannah, Jill Casey, Michael A. Ruta, Angie Mullen, Sean Waites, Elsa Ho, D ${ }^{\text {re }}$ Carol McClure, Valerie Emond, Marc Simard, Rolf Puchtinger, Shauna Démers, Marguerite Fenske, Mark Smith, Linda Van Til, Karen Tu et Joellyn Ellison.

\section{Conflits d'intérêts}

Les auteures déclarent n'avoir aucun conflit d'intérêts.

\section{Contributions des auteures}

A. F. a contribué à la revue de la littérature, à la conception de l'étude, aux analyses statistiques et à la préparation du manuscrit. L. L. a contribué à la conception de l'étude, aux analyses statistiques et à la préparation du manuscrit. K. R. a contribué à la conception de l'étude et à la préparation du manuscrit. Les trois auteures ont lu et approuvé le manuscrit final.

\section{Références}

1. Boyd CM, Fortin M. Future of multimorbidity research: how should understanding of multimorbidity inform health system design? Public Health Rev. 2010;32(2):451-474.

2. Barnett $K$, Mercer SW, Norbury M, Watt G, Wyke S, Guthrie B. Epidemiology of multimorbidity and implications for health care, research, and medical education: a cross-sectional study. Lancet. 2012;380:37-43.

3. van den Akker M, Buntinx F, Metsemakers JF, Roos S, Knottnerus JA. Multimorbidity in general practice: prevalence, incidence, and determinants of co-occurring chronic and recurrent diseases. J Clin Epidemiol. 1998;51(5):367-375.

4. Fortin M, Bravo G, Hudon C, Vanasse A, Lapointe L. Prevalence of multimorbidity among adults seen in family practice. Ann Fam Med. 2005;3(3): 223-228.

5. Rocca WA, Boyd CM, Grossardt BR, et al. Prevalence of multimorbidity in a geographically defined American population: patterns by age, sex, and race/ ethnicity [Internet]. Mayo Clin Proc. 2014;89(10):1336-1349. En ligne à : http://dx.doi.org/10.1016/j.mayocp .2014 .07 .010 
6. Koné Pefoyo AJ, Bronskill SE, Gruneir $A$, et al. The increasing burden and complexity of multimorbidity. BMC Public Health [Internet]. 2015;15:415. En ligne à : http://dx.doi.org/10.1186 /s12889-015-1733-2

7. Roberts KC, Rao DP, Bennett TL, Loukine L, Jayaraman GC. Prévalence et profils de la multimorbidité au Canada et déterminants associés. Promotion de la santé et prévention des maladies chroniques au Canada. 2015;35(6):93-101.

8. van den Akker M, Buntinx F, Roos S, Knottnerus JA. Problems in determining occurrence rates of multimorbidity. J Clin Epidemiol. 2001;54(7): 675-679.

9. Fortin M, Bravo G, Hudon C, et al. Relationship between multimorbidity and health-related quality of life of patients in primary care. Qual Life Res. 2006;15(1):83-91.

10. Salisbury C, Johnson L, Purdy S, Valderas JM, Montgomery AA. Epidemiology and impact of multimorbidity in primary care: a retrospective cohort study. Br J Gen Pract. 2011;61(582): e12-e21.

11. Wolff JL, Starfield B, Anderson G. Prevalence, expenditures, and complications of multiple chronic conditions in the elderly. Arch Intern Med. 2002; 162(20):2269-76.

12. Kuwornu JP, Lix LM, Shooshtari S. Grappes de multimorbidité chez les Autochtones et chez les non-Autochtones d'origine blanche au Canada. Maladies chroniques et blessures au Canada. 2014;34(4):238-246.

13. Agborsangaya CB, Lau D, Lahtinen M, Cooke T, Johnson JA. Multimorbidity prevalence and patterns across socioeconomic determinants: a crosssectional survey. BMC Public Health [Internet]. 2012;12(1):201. En ligne à : http://dx.doi.org/10.1186/1471-2458 $-12-201$

14. St John PD, Tyas SL, Menec V, Tate R. Multimorbidity, disability, and mortality in community-dwelling older adults. Can Fam Physician. 2014;60(5): e272-e280.
15. Statistique Canada. Chiffres de population et des logements, Canada, provinces et territoires, recensements de 2011 et 2006. [Internet]. 2015 [consultation le 21 juillet 2015]. En ligne à : http://www12.statcan.gc.ca/census -recensement/2011/dp-pd/hlt-fst /pd-pl/Table-Tableau.cfm?Lang = fra $\& \mathrm{~T}=101 \& \mathrm{~S}=50 \& \mathrm{O}=\mathrm{A}$

16. Organisation mondiale de la Santé. Classification internationale des maladies, neuvième révision. Genève $(\mathrm{CH})$ : OMS; 1977.

17. National Center for Health Statistics, Centers for Medicare and Medicaid Services. International classification of diseases, ninth revision: clinical modification (ICD 9 CM). Hyattsville (MD): National Center for Health Statistics; 1978.

18. Institut canadien d'information sur la santé. Classification statistique internationale des maladies et des problèmes de santé connexes, dixième révision. Ottawa (Ont.) : Institut canadien d'information sur la santé; 2009. En ligne à : https://www.cihi.ca/fr /icd_10_ca_vol1_2009_fr.pdf

19. Robitaille C, Bancej C, Dai S, et al. Surveillance of ischemic heart disease should include physician billing claims: population-based evidence from administrative health data across seven Canadian provinces. BMC Cardiovasc Disord [Internet]. 2013;13:88. En ligne à : http://dx.doi.org/10.1186/1471-2261 $-13-88$

20. Blais C, Dai S, Waters C, et al. Assessing the burden of hospitalized and community-care heart failure in Canada. Can J Cardiol [Internet]. 2014;30(3):352-358. En ligne à : http://dx.doi.org/10.1016/j.cjca.2013 .12 .013

21. Gershon AS, Wang C, Guan J, Vasilevska-Ristovska J, Cicutto L, To T. Identifying patients with physiciandiagnosed asthma in health administrative databases. Can Respir J [Internet]. 2009;16(6):183-8. En ligne à : https://www.ncbi.nlm.nih.gov/pmc /articles/PMC2807792/
22. Gershon AS, Wang C, Guan J, Vasilevska-Ristovska J, Cicutto L, To T. Identifying individuals with physician diagnosed COPD in health administrative databases. COPD. 2009;6(5): 388-394.

23. Kisely S, Lin E, Lesage A, et al. Use of administrative data for the surveillance of mental disorders in 5 provinces. Can J Psychiatry. 2009;54(8): 571-575.

24. Quan H, Khan N, Hemmelgarn BR, et al. Validation of a case definition to define hypertension using administrative data. Hypertension. 2009;54(6): 1423-1428.

25. Chen G, Khan N, Walker R, Quan H. Validating ICD coding algorithms for diabetes mellitus from administrative data. Diabetes Res Clin Pract [Internet]. 2010;89(2):189-95. En ligne à : http:// dx.doi.org/10.1016/j.diabres.2010.03 .007

26. Britt HC, Harrison CM, Miller GC, Knox SA. Prevalence and patterns of multimorbidity in Australia. Med J Aust. 2008;189(2):72-77.

27. SAS Institute Inc. SAS/STAT 9.3 user's guide. Cary (NC): SAS Institute Inc.; 2011.

28. Nicholson K. Multimorbidity in Canada: examining prevalence and patterns using a national electronic medical record database. Paper presented at: Society for Academic Primary Care 45th annual scientific meeting; 2016 Jul 6-8; Dublin, Ireland.

29. Fortin M, Hudon C, Haggerty J, van den Akker M, Almirall J. Prevalence estimates of multimorbidity: a comparative study of two sources. BMC Health Serv Res [Internet]. 2010 [consultation le 16 août 2016];10(111):1-6. En ligne à : http://dx.doi.org/10.1186 /1472-6963-10-111

30. Gross R, Bentur N, Elhayany A, Sherf $\mathrm{M}$, Epstein L. The validity of selfreports on chronic disease: characteristics of underreporters and implications for the planning of services. Public Health Rev. 1996;24(2):167-182. 
31. Fortin M, Stewart M, Poitras $M$, Almirall J, Maddocks H. A systematic review of prevalence studies on multimorbidity: toward a more uniform methodology. Ann Fam Med. 2012; 10(2):142-151.

32. Uijen AA, van de Lisdonk EH. Multimorbidity in primary care: prevalence and trend over the last 20 years. Eur J Gen Pract. 2008;14(Suppl 1): 28-32.

33. Wong A, Boshuizen HC, Schellevis FG, Kommer GJ, Polder JJ. Longitudinal administrative data can be used to examine multimorbidity, provided false discoveries are controlled for. J Clin Epidemiol. 2011;64(10):1109-1117.

34. Diederichs C, Berger K, Bartels DB. The measurement of multiple chronic diseases-a systematic review on existing multimorbidity indices. J Gerontol A Biol Sci Med Sci. 2011;66A(3):301-311.

35. Ladouceur M, Rahme E, Pineau CA, Joseph L. Robustness of prevalence estimates derived from misclassified data from administrative databases. Biometrics. 2007;63(1):272-279.

36. Virnig BA, McBean M. Administrative data for public health surveillance and planning. Annu Rev Public Health. 2001;22:213-230.

37. Lix LM, Walker R, Quan $\mathrm{H}$, et al. Caractéristiques des bases de données sur les services médicaux au Canada. Maladies chroniques et blessures au Canada. 2012;32(4):207-215.

38. Agence de la santé publique du Canada. Le diabète au Canada : perspective de santé publique sur les faits et chiffres. Ottawa (Ont.) : Agence de la santé publique du Canada; 2011. [no HP35-25/2011F]

39. Agence de la santé publique du Canada. Rapport du Système national de surveillance des maladies chroniques : L'hypertension au Canada, 2010. Ottawa (Ont.) : Agence de la santé publique du Canada; 2010. [no HP32-4/2010].

40. Blais C, Jean S, Sirois C, et al. Le Système intégré de surveillance des maladies chroniques du Québec (SISMACQ), une approche novatrice. Maladies chroniques et blessures au Canada. 2014;34(4):247-256. 